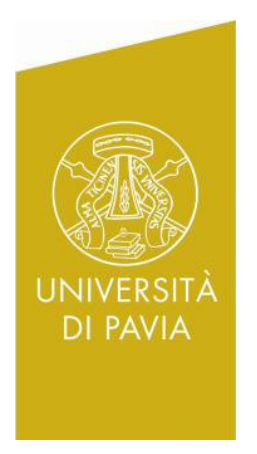

Department of Economics and Management DEM Working Paper Series

\title{
A Bayesian h-index: how to measure research impact
}

\author{
Paola Cerchiello
}

(Università di Pavia)

Paolo Giudici

(Università di Pavia)

\# 102 (05-15)

Via San Felice, 5

I-27100 Pavia

http://epmq.unipv.eu/site/home.html

May 2015 


\title{
A Bayesian h-index: how to measure research impact
}

\author{
Paola Cerchiello • Paolo Giudici
}

Received: date / Accepted: date

\begin{abstract}
The quality of academic research is difficult to measure and rather controversial. Hirsch has proposed the $h$ index, a measure that has the advantage of summarizing in a single summary statistic the information that is contained in the citation counts of each scientist. Although the $h$ index has received a great deal of interest, only a few papers have analyzed its statistical properties and implications. We claim that statistical modeling can give a lot of added value over a simple summary like the $h$ index. To show this, in the paper we propose a negative binomial distribution to jointly model the two main components of the $h$ index: the number of papers and their citations. We then propose a Bayesian model that allows to obtain posterior inferences on the parameters of the distribution and, in addition, a predictive distribution for the $h$ index itself. Such a predictive distribution can be used to compare scientists on a fairer ground, and in terms of their future contribution, rather than on their past performance.
\end{abstract}

\section{Introduction}

The quality of academic research is controversial and difficult to measure (for a discussion see, for example, [1], [3] and [17]).

Recently [14] has proposed a measure that has the advantage of summarizing in a single summary statistic the information that is contained in the citation counts of the $N_{p}$ papers published by a scientist along her/his career. Hirsch's definition is that "a scientist has index $h$ if $h$ of her/his $N_{p}$ papers have at least $h$ citations each and the other $\left(N_{p}-h\right)$ papers have $\leq h$ citations each".

Since its appearance, the $h$ index has been extensively applied to rank scientists within different communities. It has also been used to take important decisions,

\footnotetext{
P. Cerchiello

University of Pavia, Pavia, Italy

Tel.: +390382984348

Fax: +390382304226

E-mail: paola.cerchiello@unipv.it

P. Giudici

University of Pavia, Pavia, Italy
} 
such as recruitment or promotion of a scientists, depending on whether her/his $h$ index is above or below a predetermined threshold.

The widespread diffusion of the $h$ index as a quality threshold has prompted an extensive debate on its appropriateness, in comparison with other summary statistics, such as the number of papers, the number of citations, or the number of papers in high quality scientific journals. While the number of papers considers only one aspect of quality, scientific productivity, and not its impact on the society, the number of citations is a function of both and should, therefore, be considered as the main competitor of the $h$ index. Another potential competitor is the number of papers in high quality journals, although the choice of which journals is somewhat subjective.

All the above measures, including the $h$ index, exhibit a high degree of variability, arising from measurement errors. Such errors are typically related to data collection and include, for example, delays in the update of the database of papers that is used to calculate them, and linguistic homonyms, which may bias citation counts. It would be important to take this variability into account, in a proper stochastic model.

In addition, all measures depend on the seniority of a scientist: the higher the seniority, the higher the value. It would be important to compare researchers with different seniority on a fairer ground.

The original paper by Hirsch did not consider such aspects but, rather, insisted in "eliminating" variability, as it is evident from the way in which the use of the index was advocated, against the number of citations ("...which may be inflated by a small number of big hits...").

Following the seminal work of Hirsch, many papers have focused on its application, but, surprisingly, few of them have focused on the stochastic aspects behind it, apart from [12] that hinted at the relevance of a "statistical background" for the $h$ index. This, very likely, as most of the literature on the $h$ index has developed outside statistics, in the bibliometric community.

Exceptions to the above are the recent papers by [2] and [16], who have proposed an asymptotic distribution for the $h$ index, valid for highly productive researchers, that can be used for inferential purposes and not only for descriptive summaries, as the typical bibliometric contributions. From a different perspective, [18] shows how a combinatorial approach can shed light on the $h$ index.

However, very often, especially in the context of hiring and promotion decisions, the $h$ index is to be measured on scientists with a limited number of papers, either because they are at a junior level, or because they have had different duties besides research: notably, teaching and organisational ones. Thus, the assumptions behind the asymptotic approach do not hold in general, and a more general stochastic approach is needed.

The main contribution of this paper is to provide such an approach, by means of a Bayesian $h$ index. Our main claim is that proper statistical modeling can give a lot of added value over a simple summary like the $h$ index. Our basic idea is that the use of $h$ index alone is too sharp, and the use of the vector of the citations for each paper of each scientist may be employed to enrich the $h$ index in a stochastic approach that tries to take into account measurement variability and the different seniority of researchers.

More specifically, we assume that scientific quality of a scientist is a latent variable, measured by two main components: the number of produced papers and the 
corresponding citation counts vector. Such observable quantities can be modelled by means of a negative binomial distribution, that exploits, rather than eliminate, the variability present in both the production and the impact dimensions of a scientist's work. We then propose to estimate the parameters of such distribution by means of a Bayesian approach. This allows, through the implicit exchangeability assumption, the derivation of a predictive distribution for the h-index that can be used to compare scientists in a way that is less dependent on seniority and, thus, fairer.

The rest of paper is organized as follows: in section 2 we describe the proposed methodology; in section 3 we present the data to which the methodology is applied and in section 4 we describe the obtained results. In section 5 we conclude the paper with a final discussion and future research developments.

\section{The proposed Bayesian h-index}

Let $\underline{X}=\left(X_{1}, \ldots, X_{n}\right)$ be a vector of random variables representing the number of citations of the $n$ articles of a given scientist. Consider the ordered sample of citations $X_{(1)} \geq X_{(2)} \geq \ldots \geq X_{(n)}$, in which $X_{(1)}$ and $X_{(n)}$ denote the most and the least cited paper, respectively. The $h$ index can be defined as follows:

$$
h=\max \left\{t: X_{(t)} \geq t\right\} .
$$

The main advantage of the $h$ index is its simplicity as a summary measure. We claim that a proper statistical modelling of $\underline{X}$ can add considerable added value to the interpretation of the $h$ index, particularly useful when important decisions are to be taken.

An appropriate statistical model can be built exploiting the analogies with loss data modelling. Indeed, citation counts are characterised, in the same fashion as loss data, by a majority of observations with a small impact and few ones with large impact.

References to statistical models for loss data can be found in the so-called Loss Distribution Approach (see for example [9] and [10]), in which losses are categorized in terms of 'frequency' and 'severity'. The frequency is the random number of loss events occurred during a specific time frame, while the severity is the mean impact of all such events in terms of monetary losses.

In our context the frequency is the (random) number of published papers along the career of a scientist and the impact is the (random) mean number of citations received in the same time frame by all such papers. More formally, for the citation vector $\underline{X}=\left(X_{1}, \ldots, X_{n}\right)$, the two relevant quantities are $n$, the random number of published papers, and $m=\frac{\sum_{i=1}^{n} X_{i}}{n}$, the mean impact of a scientist.

In analogy with loss data modelling, we can assume that, for each scientist, the distribution of the frequency is specified by a discrete probability function $p(n \mid \lambda)$, which depends on a parameter $\lambda$, and that, conditionally on $n$, the citations of the papers, $\left(X_{i}, j=1, \ldots, n\right)$ are independent and identically distributed random variables, with a common distribution $k(x \mid \theta)$, which depends on an unknown parameter $\theta$. 
On the basis of the previous assumptions we can derive the distribution of the total number of citations of each scientist, compounding the frequency distribution with the paper citations distribution. This would be the analog of the total loss.

However, our object of interest is the $h$ index. Theoretically, we can derive its distribution from the distribution of the $X_{(i)}$ order statistics and, then, applying the definition in (1). In practice, however, such derivation is analytically unfeasible, and we should resort to Monte Carlo sampling, as follows.

Once the parameters $(\lambda, \theta)$ are estimated with $(\hat{\lambda}, \hat{\theta})$, in each simulation a value of $n$ is generated from $p(n \mid \hat{\lambda})$ and, then, $n$ values of $X_{i}$ are simulated from $k(x \mid \hat{\theta})$. From the latter the $h$ index can be calculated so that, replicating the procedure for $M$ simulations, gives an approximate distribution for it. This is the approach followed in [7].

However, the problem is that the h-index is a summary measure of the past production and impact of a scientist which can, therefore, heavily depend on individual characteristics and, in particular, on the academic age of a scientist. Longer ages obviuosly lead to higher $h$ indexes. When a scientist is evaluated, especially in terms of a possible future recruitment or promotion, the aim is to evaluate her/his possible future impact, and not the past behaviour.

To achieve this aim, we propose a Bayesian model that allows, through exchangeability, to obtain a predictive distribution of the $h$ index that can be used to fairly compare expected future performances of scientists.

More formally, consider the vector of the citations of $M$ future papers of a scientist, $\underline{Y}=\left(Y_{1}, \ldots, Y_{M}\right)$. If we assume that $\left(Y_{1}, \ldots, Y_{M}\right)$ is exchangeable, its predictive distribution can be obtained from:

$$
p\left(Y_{1}, \ldots, Y_{M}\right)=\prod_{i=1}^{M} \int p\left(y_{i} \mid \mu\right) p(\mu) d \mu,
$$

where $\mu$ is a vector of parameters, to be specified.

To specify the distribution of $\underline{Y}$, we thus need to specify the statistical model $p(x \mid \mu)$ and the prior distribution $p(\mu)$.

Concerning the statistical model, the Uniform-Poisson model proposed in [7] is not a valid assumption in a general context. It assumes that the number of produced papers is uniform, and this is an unrealistic assumption. It also assumes that citation counts are independent and identically distributed, as a Poisson distribution. However, in practice, the variance of the citations is greater than the mean, leading to overdispersion.

We suggest to improve the statistical model in [7], overcoming the weakness of both the Uniform and the Poisson distribution into a Negative Binomial compound distribution.

Specifically, the citation count of a paper of a scientist, $X_{i}$ is assumed to follow a Negative Binomial distribution, with parameters $(p, r)$ :

$$
p\left(X_{i}=k\right)=\left(\begin{array}{c}
k+r-1 \\
k
\end{array}\right)(1-p)^{k} p^{r} \quad k=0,1,2, \ldots,
$$

where, for a given scientist, $1-p$ describes the probability of success (citation) of the $i$-th paper, and $r$ is a parameter that describes the waiting time, in terms of failures (no citations), before arriving at $k$ successes. 
Note that the above distribution implies that the total number of citations of a scientist with $n$ papers is also a negative binomial, with parameters $(p, n * r)$. This distribution can also be equivalently expressed in terms of its two components: the number of papers is a Poisson with parameter equal to $\lambda=-r * \ln (1-p)$ and the citation impact of a generic paper follows a logarithmic distribution, with probability mass function equal to :

$$
\frac{(-p) * k}{k * \ln (1-p)}, k=0,1,2, \ldots
$$

Concerning the prior distribution, if we assume the previous negative binomial as a statistical model, we need to assess a prior distribution on the parameters $\mu=(p, r)$.

To ease prior elicitation and interpretation, we can consider $\mu=(1-p, r)$, with $(1-p)$ the probability of success. Furthermore, to avoid correlations between the sampled parameters in the Monte Carlo Markov chain procedure, a convenient parametrisation is to consider a function of $1-p$ :

$$
m=\frac{(1-p) * r}{p}
$$

which represents the mean number of citations (in analogy with the severity of loss models). We leave $r$ unchanged, as a parameter that determines dispersion.

Both the two unknown parameters, $m$ and $r$, are defined only on positive values and, therefore, it becomes reasonable to assign two Gamma prior distributions, respectively with (known) hyperparameters $(a, b)$ and $(c, d)$. In addition, and in analogy with loss distribution models, the two parameters can be assumed independent.

Having specified the statistical model and the prior distributions, we can derive the posterior distribution of $\mu=(m, r)$.

Such posterior distribution cannot be obtained analytically, but can be approximated by means of Markov Chain Monte Carlo sampling. The same sampling procedure can be used to derive the approximate posterior distribution of the parameters of interest, $(1-p, r)$. It can also be used to derive the approximate predictive distribution of $M$ future papers' citations, for example following the procedure in the $\mathrm{R}$ software package rjags. Consequently, the predicted value of the $h$ index, for $M$ future papers can also be obtained, applying (1).

\section{Data description}

To illustrate our proposal, we consider scientists in the field of statistics, the one that we know better, and the citation counts of their papers, published until the year 2011. The papers produced in the following years, which are nevertheless "too young" to deploy their full citation potential, will be used in a predictive performance test.

We consider two different contexts. In the first one we compare a group of internationally known top statisticians, whose $h$ indexes can be easily calculated via the ISI Web of Science, as they typically publish prevalently in top journals. This context serves as a description of our method, in terms of its implied ranking, without reference to a decision. 
In the second context we consider a group of candidates to a promotion, all belonging to the same country (Italy), with homogeneous hiring and promotion rules. This context is particularly relevant as the decision to promote a candidate or not does depends on a threshold value of the $h$ index. We now describe it in more length.

In a recent open competition for the promotion at the level of Full Professor, in Italian Universities, the Ministry of Research has fixed an entrance threshold that depends on the median $h$ index of the full professors of the considered discipline. We have calculated the $h$ index for all 131 full professors of statistics employed in Italian universities. This is indeed a small subset of the worldwide population of statisticians, but it is, on the other hand, an homogeneous cohort of people: both in terms of academic rules (they belong to the same country) and in terms of research 'modus operandi' (they belong to the same scientific community).

Among the different data sources available, we have selected the one that is most accessible, although, perhaps, of lower quality: Google Scholar. The advantage of Google Scholar is in its wide definition: it includes all sources, and not only scientific journals, published in any language, and it is updated in real time, as the source goes on-line. These advantages may be, however, a limitation, as low quality sources (such as simple web reports) may be included, along with officialy certified ones (such as scientific journals). Another possible disadvantage is that this source is based only on on-line material: a very relevant editor that is not on-line does not contribute to citations.

We have extracted a database from Google scholar that contains, for all considered professors, all their published papers, with the corresponding citation vector. The data were collected until 2011, and shortly before the opening of the competition, that has ended in 2014. We have cleaned and refined the data, in a long activity of disambiguation (from homonyms and wrong affiliations) that was carried out by employing the well known software 'Publish or Perish' ([13]).

Figure 1 describes the data in terms of number of published papers for the considered scientists.

From Figure 1 note that the distribution of the number of papers is skewed to the right. In addition, the main summary statistics from the above distribution assume the following values: mean $=35.66$, median $=30$, maximum $=128$ and minimum $=0$. These statistics are consistent with right skewness of the distribution, and reveal a rather heterogeneous publishing behaviour, in which the most productive scientist has published 128 papers and the least productive ones 0 . The fact that some professors have zero publications, although striking, can be explained by the historical evolution of recruitments in Italy and in similar countries. In the seventies, university enrollment has considerably increased. As a result, in a country that is not used to plan public spending in advance, a large intake of professors, with very limited selection criteria (especially in terms of research) have been made in the eighties. Another possible explanation is that Google Scholar considers only on-line publications: some professors, and especially the oldest, may have published papers in journals that are not on-line, or whose past issues are not.

Figure 2 describes the same data in terms of total number of citations for the considered scientists.

From Figure 2 note that the distribution of the citations is strongly skewed to the right. The main summary statistics from the above distribution assume the 


\section{Histogram of number of papers}

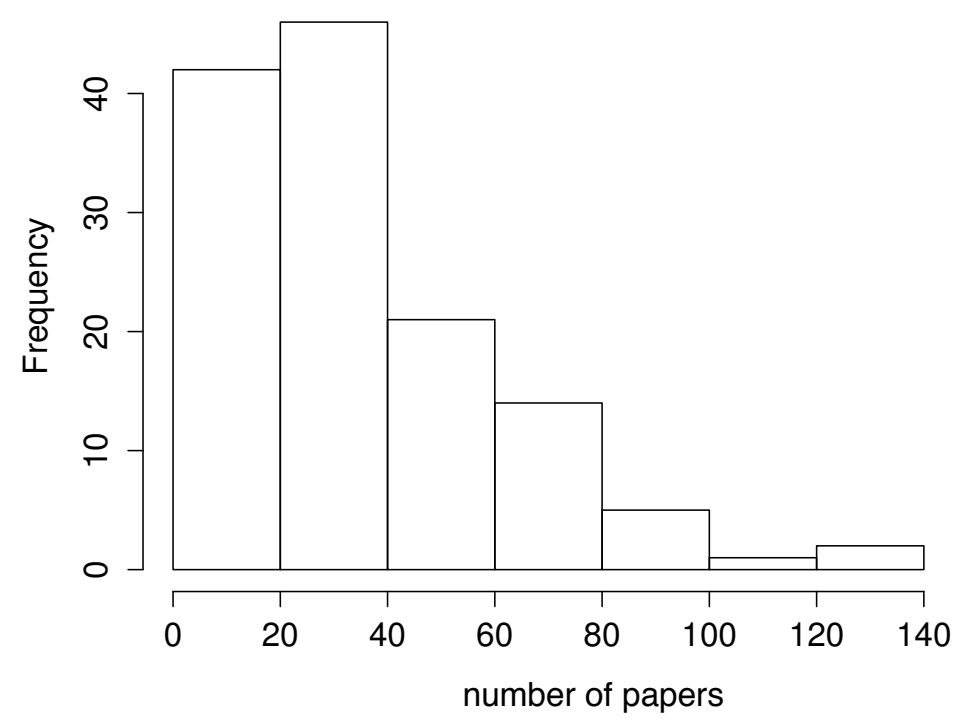

Fig. 1 Distribution of the total number of papers for each scientist

following values: mean $=206.2$, median $=107$, maximum $=2438$ and minimum $=0$. These statistics confirm that the distribution of citations is very skewed to the right, indicating a strong difference in the impact of the scientists, that ranges from 0 to 2438 total citations. Data analysis reveals that some professors have zero citations: these may be due to the same factors described commenting the zero papers case: either weak selection or papers that are not on-line.

In Figure 3 we report, for the same data, the distribution of the $h$ index.

From Figure 3 note that the distribution of the $h$ index is, as expected, similar to the distribution of the citations in Figure 2, but appears more concentrated: it has mean $=5.68$, median $=5$, maximum $=19$ and minimum $=0$. Indeed the skewness and kurtosis of the $h$ index distribution are 0.98 and 0.90 , whereas for the distributions in Figure 2 the same summary statistics are equal to 3.82 and 20.53.

On the basis of the above result, to qualify for promotion, a candidate that applies for a full professorship should have an $h$ index greater or equal than 5 (the median value of the current full professors).

In the next section we show that such a comparison may be biased, as applicants with the same threshold may exhibit rather different scientific behaviours, in the subsequent years.

We will therefore claim that promotion decisions should be based on the predictive, rather than the observed $h$ index, as the former takes into account the variability of past citations and, in addition, is less dependent on the seniority of a researcher. 


\section{Histogram of total citations}

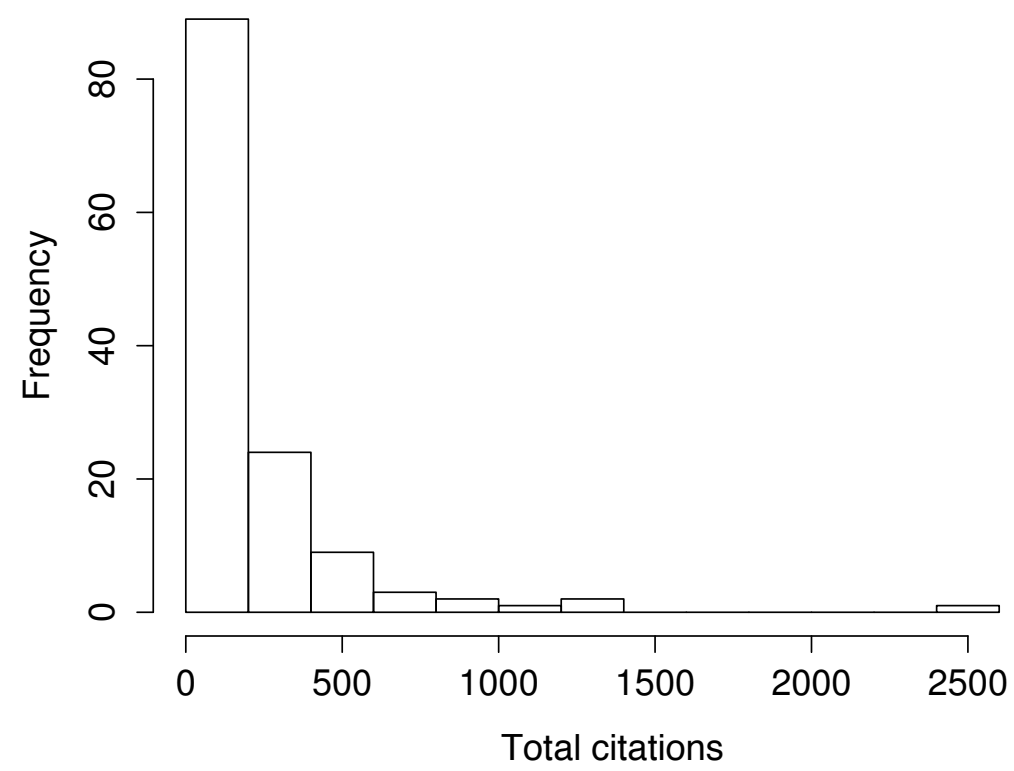

Fig. 2 Distribution of the total number of citations for each scientist

\section{The Bayesian h-index in practice}

We first calculate the predictive $h$ index for some known top statisticians, at the worldwide level: Tibshirani, Cox, Green, Efron.

Figure 4-5 show the MCMC trace plots and the corresponding approximated posterior distribution, for the parameters $p, r$, for one of the considered authors (Cox).

Figure 1 about here.

From Figure 4-5 we easily see that the algorithm has converged. The trace plots of the other scientists have a similar convergence behaviour.

Table 1 shows, for the same top statisticians, the posterior mean and standard deviations of $1-p$ and $r$.

Table 1 about here.

Table 1 Top Statisticians, estimated parameters $1-p$ and $r$

\begin{tabular}{ccccc}
\hline \hline Scholars & $1-p$ & s.d. & $r$ & s.d. \\
\hline Cox & 0.965 & 0.0003 & 0.171 & 0.011 \\
Efron & 0.995 & 0.0060 & 0.215 & 0.257 \\
Green & 0.984 & 0.0020 & 0.291 & 0.050 \\
Tibshirani & 0.998 & 0.0024 & 0.130 & 0.150 \\
\hline
\end{tabular}




\section{Histogram of $\mathrm{h}$ index}

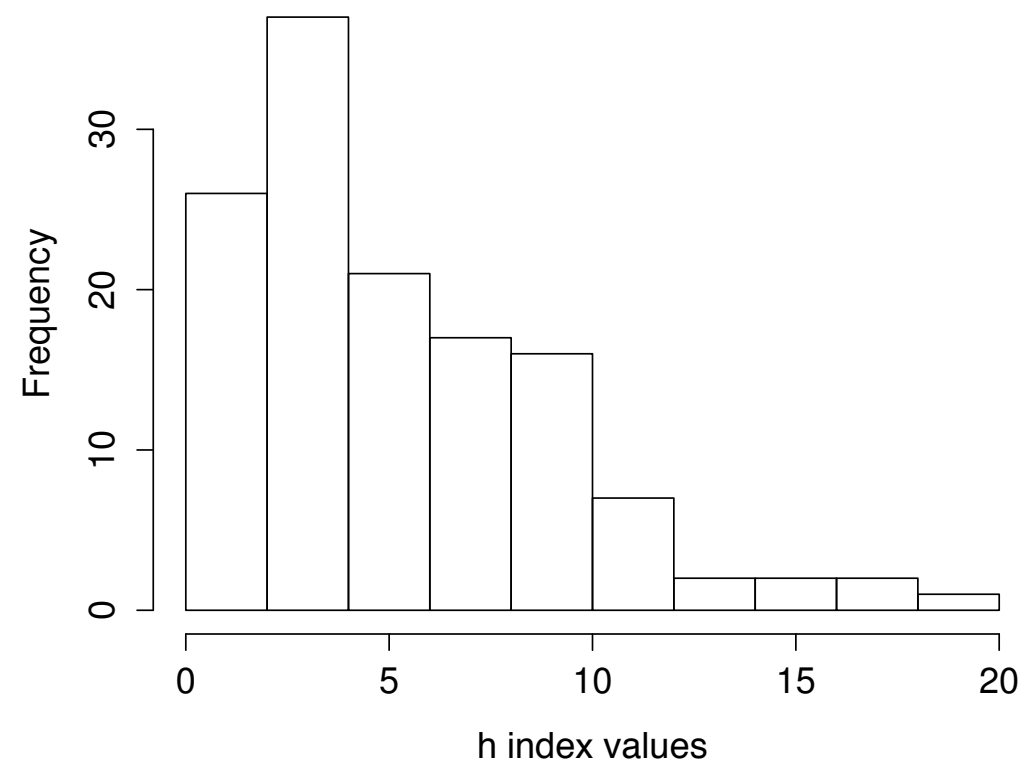

Fig. 3 Distribution of the $h$ index
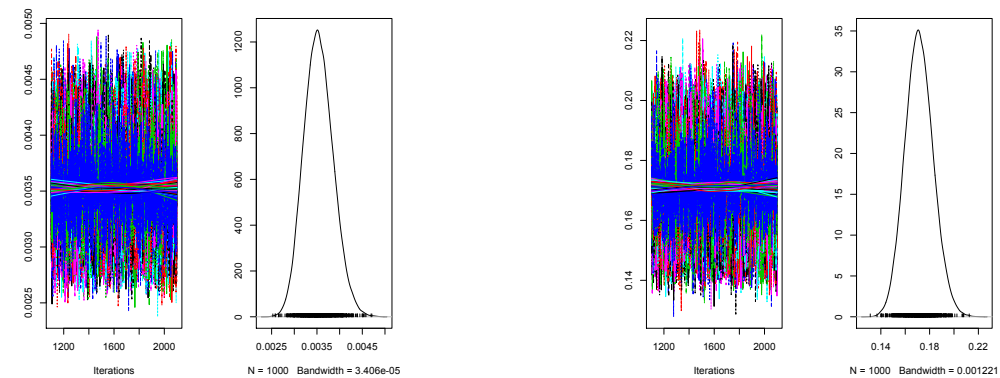

Fig. 4 Trace plot and posterior distribution forFig. 5 Trace plot and posterior distribution for Cox's $p$ parameter Cox's $r$ parameter

Table 1 show that the highest citation impact, as measured by the $1-p$ parameter, is achieved by Tibshirani, followed by Efron, Green and, finally, Cox. Moreover, Tibshirani is the most productive scientist, as it has the smallest estimated $r$, followed by Cox, with Efron and Green at some distance. The results in Table 1 indicate that Efron and Tibshirani will very likely be the most successfull scientists, in terms of impact. But also that they will have two different profiles, with Efron publishing less papers than Tibshirani. 
Table 2 shows, for the same top statisticians, the estimated values of the predictive $h$ index, along with their observed past data (1985-2011), in terms of: number of papers, total of citations, $h$ index ('85-'11), and the observed index for the years 2012-2014, that will be used to assess the performance of the predictive $h$ index.

Table 2 about here

Table 2 Top Statisticians

\begin{tabular}{cccccc}
\hline \hline Scholars & N. papers & Tot. Cit & $h$ index & Pred. $h$ index & Obs. $h$ index \\
\hline Cox & 426 & 34022 & 64 & 4.71 & 2 \\
Efron & 122 & 9527 & 45 & 5.53 & 6 \\
Green & 70 & 2248 & 25 & 4.74 & 4 \\
Tibshirani & 319 & 41924 & 68 & 4.67 & 5 \\
\hline
\end{tabular}

Table 2 shows that, among the top statisticians, Efron has, for the next $M=10$ papers, the highest predictive $h$ index, followed by the other three scientists. This is consistent with what obtained in Table 2 , and underlines that the $h$ index favors profiles with a low variability of citations, among the different papers. Indeed, the observed $h$ indexes for the subsequent 2012-2014 period show that this prediction is rather correct, as Efron has obtained a $h$ index of 6 , followed by Tibshirani, Green and Cox. Note that the top statisticians are very different in terms of productivity: Cox results to have published 426 papers, Tibshirani 319; against 122 of Efron and 60 of Green. Assuming that scientists repeat the same past productivity pattern in the future, as our predicted $h$ index is calculated for a fixed number of 10 papers, the prediction for Tibshirani may be underestimated, as it indeed appears comparing it with the observed value. The assumption of a constant productivity cannot probably be sustained for Cox, who in fact shows a considerable drop in the observed $h$ index.

We then calculate the predictive $h$ index for three Italian candidates to full professorship, as well of a top Italian full professor (in terms of $h$ index for the papers until the end of 2011). For privacy reasons the names of the candidates will be replaced with A,B,C; and that of the full professor with $\mathrm{P}$.

Figures 6-7 show the MCMC trace plots and the corresponding approximated posterior distribution, for the parameters $p, r$, for one of the considered candidates at the competition (A).

Figure 2 about here.

From Figures 6-7 we can easily see that the algorithm has converged. The trace plots of the other candidates have a similar convergence behaviour.

Table 3 shows, for the same scientists, the posterior mean and standard deviations of $1-p$ and $r$.

Table 3 about here

From Table 3 note that the highest citation impact, as measured by the $1-p$ parameter, is achieved by the Professor P, followed by candidates C, A, followed by B. Note that the citation impact of the Professor is comparable to those in Table 1, differently from those of the candidates. The candidates are, however, rather different in terms of productivity, with the estimated $r$ indicating that $\mathrm{C}$ is 

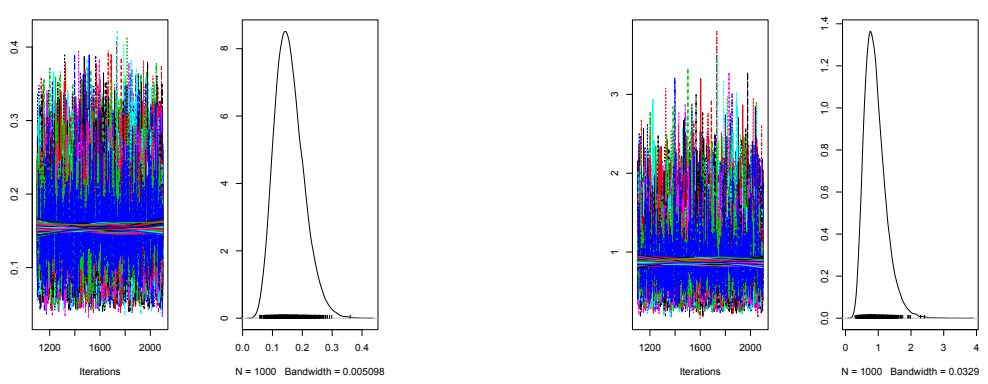

Fig. 6 Trace plot parameter $p$ of Researcher AFig. 7 Trace plot parameter $r$ of Researcher A

Table 3 Italian Statisticians, estimated parameters $1-p$ and $r$

\begin{tabular}{ccccc}
\hline \hline Scholars & $1-p$ & s.d. & $r$ & s.d. \\
\hline P & 0.972 & 0.005 & 0.195 & 0.027 \\
A & 0.844 & 0.049 & 0.912 & 0.331 \\
B & 0.828 & 0.051 & 0.655 & 0.200 \\
C & 0.934 & 0.024 & 0.393 & 0.174 \\
\hline
\end{tabular}

the most productive, followed by B and A. The distance with the Professor and with the top scientists appears considerably higher than in terms of impact.

The results in Table 3 indicate that, assuming a similar future career length, $\mathrm{C}$ and A will very likely be the most successfull scientists, in terms of impact. However, $\mathrm{C}$ is more productive than $\mathrm{A}$, in the similar fashion as Efron is more productive than Tibshirani.

Table 4 shows, for the same scientists, the estimated values of the predicted h-index, along with their observed past data (1985-2011), in terms of: number of papers, total of citations, $h$ index, as well as their years of seniority and the observed index for the years 2012-2014, that will be used to assess the performance of the predicted $h$ index.

Table 4 about here.

Table 4 Italian Statisticians

\begin{tabular}{ccccccc}
\hline \hline Scholars & N. papers & Tot. Cit & $h$ index & Seniority & Pred. $h$ index & Obs. $h$ index \\
\hline P & 101 & 1210 & 17 & 20 & 3.91 & 4 \\
A & 18 & 112 & 5 & 13 & 4.09 & 4 \\
B & 36 & 112 & 5 & 12 & 3.14 & 3 \\
C & 11 & 135 & 5 & 25 & 3.64 & 2 \\
\hline
\end{tabular}

From Table 4 note that, consistently with the findings in Table 3 , the three candidates, who have the same (past) $h$ index of 5 , have different values of the predicted $h$ indexes. While candidates $\mathrm{A}$ and $\mathrm{B}$ maintain high values, comparable with that of the top professor candidate $\mathrm{C}$ is at much lower levels. 
In terms of performance of the predictions note that, apart from candidate C, who very likely has a non constant production path (similarly to Cox), the predictive and the observed $h$ index are quite close. This regardless of the fact that all candidates have published less than 10 papers in the period 2012-2014.

\section{Conclusions}

In this paper we have addressed the topic of correctly measuring quality of scientific research, taking statistical variability into proper account. To achieve this task, we have embedded the citation counts, of which the $h$ index is a function, in an appropriate probability framework that takes inspiration from loss data modeling. Our additional methodological contribution is to employ a citation distribution that takes overdispersion into account and a Bayesian modelling framework that allows the derivation of a predictive $h$ index.

The application of the methodology shows that our proposed Bayesian $h$ index can boost the descriptive power of the measure proposed by Hirsch, taking citations variability into proper account, and allowing a fairer comparison between scientists of different seniority.

We remark that our approach can be applied, without loss of generality, not only to compare scientists but also to compare different communities of scientists, in terms of universities, research departments, age groups, career groups, and so on.

Moreover, our proposal can be applied to other contexts, where the key statistical elements are a random frequency and a corresponding number of random discrete severities. This is the case for the evaluation of the quality of financial tweets, as in the paper by $([8])$.

Acknowledgements The authors thank the editor and the referees for very useful comments The authors acknowledge financial support from the PRIN Misura project. .

\section{References}

1. Adler, R., Ewing, J. and Taylor, P. (2009). Citation statistics: A report from the international mathematical union (IMU) in cooperation with the international council of industrial and applied mathematics (ICIAM) and the institute of mathematical statistics (IMS). Statistical Science 24 pp. 114.

2. Beirlant, J. and Einmahl, J. H. J. , Asymptotics for the Hirsch Index: Scandinavian Journal of Statistics, 37, 355-364, 2010.

3. Bornmann, L., Mutz, R. and Daniel, H.-D. (2010). The h index research output measurement: Two approaches to enhance its accuracy. Journal of Informetrics 4407414.

4. Burrell, Q. L., Hirsch's h-index: A stochastic model, Journal of Informetrics, 1, 16-25, 2007.

5. Bohlmann, H., Mathematical Methods in Risk Theory, Grundlehrenband 172, SpringerVerlag, Heidelberg, 1970.

6. Cerchiello, P. and Giudici, P., On the distribution of functionals of discrete ordinal variables: Statistics \& Probability Letters, 82, 2044-2049, 2012.

7. Cerchiello, P. and Giudici, P., H-index: a statistical proposal. Scientometrics, 99, 299-312, 2014.

8. Cerchiello, P., and P. Giudici,How to measure the quality of financial tweets. Forthcoming in Quality \& Quantity Journal.

9. Cruz, M. G., Modeling, measuring and hedging operational risk, Wiley 2002. 
10. Dalla Valle, L. and Giudici, P., A Bayesian approach to estimate the marginal loss distributions in operational risk management, Computational Statistics \& Data Analysis, 52, 3107-3127, 2008

11. Frachot, A., Moudoulaud, O. and Roncalli, T., Loss distribution approach in practice, In The Basel Handbook, A Guide for Financial Practitioners, ed. M. K. Ong, Risk Books, London, 2004.

12. Glanzel, W., On the h-index - A mathematical approach to a new measure of publication activity and citation impact, Scientometrics, 67, 315-321, 2006.

13. Harzing, A.W., Publish or Perish, available from http://www.harzing.com/pop.htm, 2007.

14. Hirsch, J. E., An index to quantify an individual's scientific research output: Proceedings of the National Academy of Sciences of the United States of America, v. 102, p. 16569-16572, (2005).

15. Iglesias, J. E. and Pecharroman C., Scaling the h-index for different scientific ISI fields: Scientometrics, 73, 303-320, 2007.

16. Pratelli, L., Baccini, A., Barabesi, L. and Marcheselli, M., Statistical Analysis of the Hirsch Index, Scandinavian Journal of Statistics, 39, 681-694, 2012.

17. Redner, S., On the meaning of the h-index, Journal of Statistical Mechanics: Theory and Experiment 2010.03 (2010): L03005.

18. Yong, A., Critique of Hirschs Citation Index: A Combinatorial Fermi Problem." arXiv preprint arXiv:1402.4357 (2014). 